\title{
High prevalence and lack of diversity of Wolbachia pipientis in Aedes albopictus populations from Northeast Brazil
}

\author{
Alessandra Lima de Albuquerque, Tereza Magalhães, Constância Flávia Junqueira Ayres/+ \\ ${ }^{1}$ Departamento de Entomologia, Centro de Pesquisas Aggeu Magalhães-Fiocruz, Av. Moraes Rego s/n, 50670-420 Recife, PE, Brasil
}

\begin{abstract}
The use of Wolbachia as a tool to control insect vectors has recently been suggested. In this context, studies on the prevalence and diversity of this bacterium in wild populations are relevant. Here, we evaluated the diversity of two Wolbachia genes (ftsZ and wsp) and the prevalence of this endosymbiont in wild Aedes albopictus. Using seminested polymerase chain reaction, our results showed that 99.3\% of the individuals were superinfected with Wolbachia. In regards to genetic diversity, the two genes showed no variation within or among mosquito populations. An analysis of other Wolbachia markers may help to clarify the relationship between insect and endosymbiont.
\end{abstract}

Key words: endosymbiont - mosquito control - semi-nested PCR

Wolbachia pipientis is an intracellular bacterium belonging to the Anaplasmataceae family (Scola et al. 2005 ) that infects a broad range of insects in which infection rates vary from $16-66 \%$ of the species screened (Hilgenboecker et al. 2008). Part of the success of the prevalence of Wolbachia in these organisms may be explained by the mode of dispersion. Wolbachia are spread through the manipulation of reproductive processes in arthropods, with cytoplasmic incompatibility (CI) as the most common phenotype. CI occurs when non-infected females cannot produce offspring when they mate with Wolbachia-infected males, thereby privileging Wolbachia-infected females as these will produce offspring when mating with infected males (Hoffmann 2005).

A majority of insects harbour one of the two clades (or supergroups) of $W$. pipientis, $\mathrm{A}$ or $\mathrm{B}$, which are found exclusively in arthropods (Werren et al. 2008). However, multiple infections have been observed in a variety of insect species, including Aedes albopictus, thus making the CI process more complex as individuals infected with different Wolbachia strains are not compatible for mating (Sinkins 2004).

Superinfection, defined by infection with two or more Wolbachia strains, allows genetic exchange to occur among supergroups, thereby increasing bacteria diversity (Werren et al. 1995, Jiggins et al. 2001). Recombination events in $W$. pipientis permit the use of these bacteria as a tool to genetically manipulate insect populations by introducing genes of interest into the $W$. pipientis genome (Werren \& Bartos 2001).

For these reasons, the use of Wolbachia as a tool for driving genes has become a subject of interest among the scientific community, considering its potential application

Financial support: CNPq, FACEPE (APQ 1361-4.00/08)

+Corresponding author: tans@cpqam.fiocruz.br

Received 23 February 2011

Accepted 9 August 2011 to control agricultural pests and insect vectors (Bourtzis 2008). Other control strategies include the utilisation of Wolbachia strains that shorten insect longevity (wMelPop) (Moreira et al. 2009). Field trials to release Aedes aegypti infected with wMelPop Wolbachia strain are scheduled for 2011 in Australia (Popovici et al. 2010).

$A$. albopictus is among the medical important mosquitoes that are naturally infected with Wolbachia (Zhou et al. 1998). Although A. albopictus has not been associated with dengue epidemics in Brazil, larvae naturally infected with dengue virus have been found in the state of Minas Gerais (MG) (Serufo et al. 1993). Moreover, this mosquito has been considered the main vector in dengue epidemics in Japan, Indonesia, Seychelles, Thailand, Malaysia (Hawley 1988) and Hawaii (Effler et al. 2005).

In the present study, the prevalence and diversity of $W$. pipientis in wild $A$. albopictus from Brazil were studied through the analysis of two single copies $W$. pipientis chromosomal genes, ftsZ and wsp (Werren et al. 1995, Braig et al. 1998). ftsZ is a cell cycle gene involved in the regulation of cell division (Werren et al. 1995). wsp codes for a major Wolbachia surface protein (Braig et al. 1998) and is considered a quickly evolving gene (Zhou et al. 1998). The maximum divergence of wsp sequences among strains that infect distinct insect taxa is $16 \%$ for supergroup A, 23\% for supergroup B and 23\% between A-B (Zhou et al. 1998, Van Meer et al. 1999); ftsZ diverged $3 \%, 6 \%$ and $15 \%$ for strains $\mathrm{A}, \mathrm{B}$ and between A-B, respectively (Werren et al. 1995). We did not find any bibliography on ftsZ and wsp diversity among $A$. albopictus within the same population (intrapopulation analysis).

Previous analysis of $f t s Z$ and wsp suggest that recombination events are common features for Wolbachia evolution and that superinfection plays an important role in recombination. However, nothing is known about the coinfection rate necessary for recombination (Werren \& Bartos 2001, Jiggins 2002, Baldo et al. 2006). If Wolbachia is to be used for vector population control through CI, the identification of potentially new strains within the same supergroup is important as these strains may also cause $\mathrm{CI}$. 
Considering the interest of using Wolbachia as a genetic drive tool or as a controlling agent of insect vectors, information about its prevalence and/or diversity in natural insect populations is extremely relevant, nevertheless it is scarce. In this study, the prevalence and diversity of two Wolbachia genes in A. albopictus from Northeast Brazil were analysed.

To analyse the prevalence of W. pipientis in A. albopictus, 150 mosquitoes from three neighbourhoods (Dois Irmãos, Engenho do Meio and Morro da Conceição) of Recife, state of Pernambuco (PE), Brazil, were assayed. These mosquitoes were obtained from eggs collected through installed ovitraps similar to the model developed by Fay and Perry (1965). Eggs were hatched and larvae were maintained until the adult phase in the insectary of the Department of Entomology at Aggeu Magalhães Research Centre-Oswaldo Cruz Foundation. Adult mosquitoes were separated by sex and total DNA was individually extracted according to the protocol described by Ayres et al. (2002).

For the analysis of $W$. pipientis diversity, 20 mosquitoes from each of the above mentioned neighbourhoods and samples from the Culicidae DNA bank of the Department of Entomology were used. Samples from the DNA bank consisted of four individuals from other neighbourhoods of Recife (Parnamirim, Casa Forte and Dois Unidos), eight individuals from other cities of PE (Olinda, Zona Rural and Bultrins neighbourhood; Moreno, Cohab and Bonança), and a Lab strain representative of Moreno neighbourhood. In order to check if different A. albopictus populations harboured different Wolbachia strains, mosquitoes from other states (also obtained from the Culicidae DNA bank) were also included in the analysis: two individuals from Rio de Janeiro (city of Rio de Janeiro, Manguinhos and Jacarepaguá neighbourhoods) and one from MG (city of Passos).

Initially, diagnosis of $W$. pipientis infection in the 150 A. albopictus individuals was performed through standard polymerase chain reaction (PCR) using primers that amplify a fragment of $432 \mathrm{bp}$ of the $f t s Z$ gene ( $W$. pipientis supergroup-specific), as described by Baldo et al. (2006). As a positive control, DNA from an individual mosquito previously diagnosed with $W$. pipientis A and B supergroups was utilised. As a negative control, no DNA was added to the PCR reaction. Samples that were negative for $W$. pipientis were assayed in a second PCR reaction with primers that amplify a region of $122 \mathrm{bp}$ of the ribosomal gene rpl8 (Lan \& Fallon 1992). This was used as an endogenous control to check if the negative result was due to a lack of or poor quality DNA, or to the absence of $W$. pipientis. Samples that were not amplified by the rpl8 primers were excluded from analysis.

In order to increase the sensitivity of Wolbachia detection, the samples that were negative by standard PCR (except the $r p l 8$ negatives) and infected samples that were diagnosed with only one strain of Wolbachia were further analysed by semi-nested PCR assays. The general Wolbachia-specific primers, wsp $81 \mathrm{~F}$ and wsp691R (618$632 \mathrm{bp}$ ), were used in the first PCR and $0.5 \mu \mathrm{L}$ of the PCR product was used in the second PCR (semi-nested PCR) utilising the primers $w s p 136 \mathrm{~F}$ and $w \operatorname{sp} 691 \mathrm{R}(577 \mathrm{bp})$ to detect supergroup A and wsp $81 \mathrm{~F}$ and $w s p 522 \mathrm{R}(449 \mathrm{bp})$ to detect supergroup B. These primers have been previously described according to Wolbachia classification to discriminate supergroups A and B (Zhou et al. 1998).

Samples utilised for $W$. pipientis genetic diversity analysis (cited above) had been previously diagnosed with both W. pipientis strains (A and B). Diversity was assessed by analysing fragments of $f t s Z$ and $w s p$ genes. Primers for fts $Z$ and $w s p$ were described above. PCR products were purified by GFX PCR DNA and Gel Band Purification Kit (GE Healthcare) then sequenced from both directions. Bioinformatics analyses were performed through CodonCode Aligner version 3.6.1 (to form contigs and to exclude poor quality sequences) and BioEdit/ClustalW (Hall 1999) for multiple alignment and manual editing. Sequence identity was confirmed by Blast search.

For comparative analysis of Wolbachia infection among populations, chi-square analyses were performed in addition to Fisher's exact tests. In all cases, 5\% was considered a significant level. The software programs utilised were Excel 2000 and R v2.10.0. (Zar 1996).

This is the first published study to investigate Wolbachia prevalence and its genetic diversity in mosquitoes from Brazil. Out of the 150 A. albopictus tested, seven were excluded from analysis as the rpl8 gene (endogenous control) was not amplified. Of the remaining 143 samples screened through standard PCR, 91.61\% were PCR positive for Wolbachia and 67.13\% (96/143) were superinfected with both strains [37.9\% of males $(48 / 58)$ and $87.1 \%$ of females $(83 / 85)$ ]. There was a significant difference between the percentages of infected males versus females; $82.76 \%$ of males and $97.65 \%$ of females were infected $(\mathrm{p}=0.0043)$.

The detection of an unexpected number of negative samples or individuals harbouring only one Wolbachia strain in our study, in both males and females, led us to use a second method to confirm this observation. In a study conducted in A. albopictus by Kittayapong et al. (2002a), the confirmation of Wolbachia infection status was carried out by a PCR screen of F1 from field collected females. However, two facts raised issues about the F1 screening method: (i) males do not transmit the bacteria to the next generation, thus Wolbachia prevalence in males cannot be performed through this method and (ii) females may present imperfect maternal transmission and only pass a single strain to their offspring, as shown by Kittayapong et al. (2002b). Thus, the Wolbachia infection data shown in the present study were initially obtained by traditional PCR and then through semi-nested PCR of negative samples and those diagnosed with a single strain. We were not able to compare the prevalence rate in males found here to other studies as no literature on the frequency of Wolbachia in males was found.

According to Berticat et al. (2002), Wolbachia density may vary between females and males, with a lower density in males. If this was true for samples analysed here, it may partially explain the lower superinfection rate in males by standard PCR as there is a DNA threshold in the template to allow amplification by PCR in comparison to nested or long PCR (Dutton \& Sinkins 2004), i.e., if the amount of DNA is too low in a sample, 
there may be no amplification, leading to a false negative result. Wolbachia density could also be influenced by environmental changes. Previous studies showed that changes, such as a long exposure to organophosphates (which can affect fitness cost), may interfere with $\mathrm{Wol}$ bachia density (Berticat et al. 2002).

When these samples were re-examined by semi-nested PCR, the values of Wolbachia prevalence changed in all cases. For instance, $99.3 \%$ of the 143 individuals were superinfected with both Wolbachia strains, differing significantly from the $67.13 \%$ obtained by standard PCR $(p=0.000)$. Males showed superinfection in $100 \%$ of the samples. Using this method, no mosquito was negative for Wolbachia (superinfected or with 1 strain) and only one female from Engenho do Meio neighbourhood (1.18\%) was diagnosed with only one strain (B). In regards to the relationship between infected males and females, the semi-nested PCR did not show any significant difference $(\mathrm{p}=1)$.

Our results showed that $98.82 \%$ of A. albopictus females were superinfected with $W$. pipientis A and B strains, similar to what was found in A. albopictus females from Thailand by Kittayapong et al. (2002b) ( $97.5 \% ; \mathrm{p}=0.7475$, compared to $98.82 \%$ found here) and Kittayapong et al. (2002a) $(97.93 \%$; $\mathrm{p}=0.8728$, compared to $98.82 \%$ found here). The general data obtained with semi-nested PCR is corroborated by studies showing that Wolbachia superinfection in A. albopictus females is a common and stable event (Kittayapong et al. 2002a, Sinkins 2004). Moreover, the semi-nested PCR-based method seemed to be efficient for surveying Wolbachia prevalence and is recommended for further screening of negative individuals that were diagnosed by traditional PCR.

According to the quality criteria of the CodonCode Aligner program (quality value $\geq 20$, error probability for the base call $\leq 1 \%$ ), 70 sequences of $f t s Z$ A, 65 of fts $Z \mathrm{~B}, 40$ of $w s p \mathrm{~A}$ and 71 of $w s p \mathrm{~B}$ genes were chosen for analysis from the $14 \mathrm{~A}$. albopictus populations. The results showed there was no variation in the nucleotide sequences among individuals within the same population (intrapopulation) or among different populations (inter-population). The nucleotide sequences of $w s p$ and $f t s Z$ were $100 \%$ identical to those published by Werren et al. (1995), Zhou et al. (1998) and Baldo et al. (2006).

Similar to the results presented here, Armbruster et al. (2003) found no variation when they analysed wsp sequences in $18 \mathrm{~A}$. albopictus individuals collected in 14 regions distributed throughout the new and old world. However, in the Armbruster et al. (2003) study, the lack of diversity could be the result of the sampling methodology (only 1 individual per locality). On the other hand, Reuter and Keller (2003) observed a high recombination rate of wsp among Formica exsecta individuals from the same population that was superinfected by five Wolbachia strains, of which three may have arisen from homologous recombination. As for the ftsZ gene, our findings showed no divergence, while in another study six different sequences were found among 11 populations of the spider Hylyphantes graminicola collected from distinct geographic regions (Yun et al. 2010).
Ayres et al. (2002) found high genetic variability and divergence among A. albopictus populations from different regions of Brazil. Notwithstanding, the results presented here and in other studies (Armbruster et al. 2003) indicate that Wolbachia strains infecting A. albopictus are stable and highly conserved, independent of the degree of divergence among mosquito populations. Two hypotheses that may explain the conservation of $f t s Z$ and wsp in Wolbachia infecting A. albopictus are: (i) there is probably a lower density of one strain compared to the other (Dutton \& Sinkins 2004), thereby decreasing the chances of homologous recombination or (ii) these genes may have a possible role in the CI phenotype, causing a high selective pressure that may inhibit the emergence of new strains. Clearly, these hypotheses must be further studied. In addition, analysis of other Wolbachia markers, such as intergenic sites (Petridis \& Chatzidimitriou 2011), may help to clarify the symbiotic relationship between these bacteria and their hosts, thereby aiding the development of novel mosquito control strategies.

\section{ACKNOWLEDGEMENTS}

To PDTIS/Fiocruz, for using its facilities, and to George Tadeu, for his help in analyzing the data.

\section{REFERENCES}

Armbruster P, Damsky WE Jr, Giordano R, Birungi J, Munstermann LE, Conn JE 2003. Infection of New and Old-World Aedes albopictus (Diptera: Culicidae) by the intracellular parasite Wolbachia: implications for host mitochondrial DNA evolution. J Med Entomol 40: 356-360.

Ayres CFJ, Romao TPA, Melo-Santos MAV, Furtado AF 2002. Genetic diversity in Brazilian populations of Aedes albopictus. Mem Inst Oswaldo Cruz 97: 871-875.

Baldo L, Dunning Hotopp JC, Jolley KA, Bordenstein SR, Biber SA, Choudhury RR, Hayashi C, Maiden MC, Tettelin H, Werren JH 2006. Multilocus sequence typing system for the endosymbiont Wolbachia pipientis. Appl Environ Microbiol 72: 7098-7110.

Berticat C, Rousset F, Raymond M, Berthomieu A, Weill M 2002. High Wolbachia density in insecticide-resistant mosquitoes. Proc Biol Sci 269: 1413-1416.

Bourtzis K 2008. Wolbachia-based technologies for insect pest population control. Adv Exp Med Biol 627: 104-113.

Braig HR, Zhou W, Dobson SL, O’Neill SL 1998. Cloning and characterization of a gene encoding the major surface protein of the bacterial endosymbiont Wolbachia pipientis. J Bacteriol 180: 2373-2378.

Dutton TJ, Sinkins SP 2004. Strain-specific quantification of Wolbachia density in Aedes albopictus and effects of larval rearing conditions. Insect Mol Biol 13: 317-322.

Effler PV, Pang L, Kitsutani P, Vorndam V, Nakata M, Ayers T, Elm J, Tom T, Reiter P, Rigau-Perez JG, Hayes JM, Mills K, Napier M, Clark GG, Gubler DJ 2005. Dengue fever, Hawaii, 2001-2002. Emerg Infect Dis 11: 742-749.

Fay RW, Perry AS 1965. Laboratory studies of ovipositional preferences hatch of Aedes aegypti. Mosq News 25: 276-281.

Jiggins FM 2002. The rate of recombination in Wolbachia bacteria. Mol Biol Evol 19: 1640-1643.

Jiggins FM, von Der Schulenburg JH, Hurst GD, Majerus ME 2001. Recombination confounds interpretations of Wolbachia evolution. Proc Biol Sci 268: 1423-1427. 
Hall TA 1999. BioEdit: a user-friendly biological sequence alignment editor and analysis program for Windows 95/98/NT. Nucleic Acids Symp Ser 41: 95-98.

Hawley WA 1988. The biology of Aedes albopictus. J Am Mosq Control Assoc 1 (Suppl. 1): 1-39.

Hilgenboecker K, Hammerstein P, Schlattmann P, Telschow A, Werren JH 2008. How many species are infected with Wolbachia? A statistical analysis of current data. FEMS Microbiol Lett 281: 215-220.

Hoffmann AA 2005. Entomology: incompatible mosquitoes. Nature 436: 189.

Kittayapong P, Baimai V, O'Neill SL 2002a. Field prevalence of Wolbachia in the mosquito vector Aedes albopictus. Am J Trop Med Hyg 66: 108-111.

Kittayapong P, Baisley KJ, Sharpe RG, Baimai V, O’Neill SL 2002b. Maternal transmission efficiency of Wolbachia superinfections in Aedes albopictus populations in Thailand. Am J Trop Med Hyg 66: 103-107.

Lan Q, Fallon AM 1992. Sequence analysis of a mosquito ribosomal protein $r p L 8$ gene and its upstream regulatory region. Insect $\mathrm{Mol}$ Biol 1: 71-80.

Moreira LA, Iturbe-Ormaetxe I, Jeffery JA, Lu G, Pyke AT, Hedges LM, Rocha BC, Hall-Mendelin S, Day A, Riegler M, Hugo LE, Johnson KN, Kay BH, McGraw EA, van den Hurk AF, Ryan PA, O'Neill SL 2009. A Wolbachia symbiont in Aedes aegypti limits infection with dengue, Chikungunya, and Plasmodium. Cell 139: 1268-1278.

Petridis M, Chatzidimitriou D 2011. Characterization of an intergenic polymorphic site (pp-hC1A_5) in Wolbachia pipientis (wPip). Mol Ecol Resour 11: 753-756.

Popovici J, Moreira LA, Poinsignon A, Iturbe-Ormaetxe I, McNaughton D, O’Neill SL 2010. Assessing key safety concerns of a Wolbachia-based strategy to control dengue transmission by Aedes mosquitoes. Mem Inst Oswaldo Cruz 105: 957-964.
Reuter M, Keller L 2003. High levels of multiple Wolbachia infection and recombination in the ant Formica exsecta. Mol Biol Evol 20: 748-753.

Scola B, Bandi C, Raoult D 2005. Genus IV. Wolbachia Hertig 1936, 472 AL. In DJ Brenner, NR Krieg, GM Garrity, JT Staley, DR Boone, P Vos, M Goodfellow, FA Rainey, K-H Schleifer (eds.), Bergey's manual ${ }^{\circledR}$ of systematic bacteriology, Springer, New York, p. 138-143.

Serufo JC, de Oca HM, Tavares VA, Souza AM, Rosa RV, Jamal MC, Lemos JR, Oliveira MA, Nogueira RMR, Schatzmayr HG 1993. Isolation of dengue virus type 1 from larvae of Aedes albopictus in Campos Altos city, state of Minas Gerais, Brazil. Mem Inst Oswaldo Cruz 88: 503-504.

Sinkins SP 2004. Wolbachia and cytoplasmic incompatibility in mosquitoes. Insect Biochem Mol Biol 34: 723-729.

Van Meer MM, Witteveldt J, Stouthamer R 1999. Phylogeny of the arthropod endosymbiont Wolbachia based on the wsp gene. Insect Mol Biol 8: 399-408.

Werren JH, Baldo L, Clark ME 2008. Wolbachia: master manipulators of invertebrate biology. Nat Rev Microbiol 6: 741-751.

Werren JH, Bartos JD 2001. Recombination in Wolbachia. Curr Biol 11: 431-435.

Werren JH, Zhang W, Guo LR 1995. Evolution and phylogeny of Wolbachia: reproductive parasites of arthropods. Proc Biol Sci 261: 55-63.

Yun Y, Lei C, Peng Y, Liu F, Chen J, Chen L 2010. Wolbachia strains typing in different geographic population spider. Hylyphantes graminicola (Linyphiidae). Curr Microbiol 62: 139-145.

Zar JH 1996. Biostatistical analysis, 3rd ed., Prentice Hall, New Jersey, $662 \mathrm{pp}$.

Zhou W, Rousset F, O'Neil S 1998. Phylogeny and PCR-based classification of Wolbachia strains using wsp gene sequences. Proc Biol Sci 265: 509-515. 